\title{
MORBILIDAD DE LA MUJER TRABAJADORA, SERVICIO DE SALUD CONCEPCION, CHILE
}

\author{
MORBIDITY IN WORKING WOMAN, CONCEPCION HEALTH SERVICE \\ JURISDICTION, CHILE
}

\author{
ALEXANDRA TORRESAGUAYO* y TATIANA PARAVIC KLIJN**
}

\begin{abstract}
RESUMEN
Estudio de tipo descriptivo y correlacional, cuyo objetivo general fue conocer las causas de morbilidad de las mujeres trabajadoras que residen en el área de jurisdicción del Servicio de Salud Concepción y analizar las diferencias existentes según características personales y de trabajo. Para efectos del estudio, se consideró la totalidad de las mujeres trabajadoras que duranteel año 2003 hicieron uso delicencias médicas por morbilidad, con un total de 28.750 licencias analizadas. Se identificaron como principales grupos de causas de morbilidad los trastornos del comportamiento con altas tasas de síndromes depresivos y ansiosos, seguidos en magnitud por las alteraciones osteomusculares donde los lumbagos y las enfermedades cervicobraquiales tienen la mayor tasa de incidencia. Ambos grupos representaron el $45 \%$ de la morbilidad laboral. Se observó que la asociación morbilidad laboral y edad es común a todas las edades y está directamente relacionada con la actividad laboral donde se desempeña la mujer trabajadora. Este hallazgo evidencia la visión reduccionista del trabajo y salud. Las causas encontradas son catalogadas como enfermedades comunes y en donde el análisis de causas hace evidenteel peso que tiene el componente social y el rol de la mujer en nuestra sociedad.
\end{abstract}

Palabras claves: M orbilidad, mujer, trabajadora.

\begin{abstract}
This is a descriptiveand correlation study. Its general objective is to know the causes of morbidity of the working women who reside in the area of jurisdiction of the Health Service of Concepción and to analyze the existing differences according to personal characteristic and work. To achieve this, it has been considered the totality of the working women who during the year 2003 have used medical licenses for morbidity with a result of 28.750 analyzed licenses. The behavior disorders were identified as principal group of morbidity causes by high rates of depression and anxiety, followed in magnitude by the osteomuscular alterations where the lumbagoes and the cervicobrachial diseases have higher rate of incidence. Both groups represented $45 \%$ of the job morbidity. It was found that the association job morbidity and age, is common to all the ages and it is directly related to the job activity of the working women. These results indicate the reduccionist vision of the work and health; the causes are catalogued as common diseases and where the analysis of causes makes evident the weight that the social component and the role of the woman in our society has.
\end{abstract}

Keywords: M orbidity, woman, worker.

Recepción: 22.12.2004. Aceptación: 21.04.2005.

*Enfermera. Licenciada en Epidemiología, Magister en Bioestadística Universidad deChile. Doctoranda Programa Doctorado en Enfermería, Universidad de Concepción, Concepción, Chile. Docente Universidad San Sebastián, Concepción, Chile. E-mail: atorres@ssconcepcion.cl

**Enfermera. Magíster en Enfermería en Salud Comunitaria, Universidad de Concepción. Doctora en Enfermería, Universidad de Sao Paulo RP, Brasil. Profesora Titular, Departamento de Enfermería, Facultad de Medicina, Universidad de Concepción, Concepción, Chile. E-mail: tparavic@udec.cl 


\section{INTRODUCCIÓN}

Durantelasúltimas décadas, en Chileal igual que en el resto de Latinoamérica, se han producido importantes transformaciones económicas y sociales, algunas de los cuales se asocian con cambios positivos en la situación de las mujeres, destacando entre ellos el mayor reconocimiento dela jefatura dehogar femenina en hogares biparenterales, el descenso de la tasa de fecundidad y la creciente participación de las mujeres en el mundo laboral (Bravo, 2004), todo lo cual le ha permitido gozar demayor autonomía, el fortalecimiento desu identidad y autoestima personal.

No obstante, esta fuerte incorporación de las mujeres al mercado laboral ha traído consigo múltiples repercusiones, no sólo en el funcionamiento familiar y laboral, sino también en las condiciones de salud y bienestar de la mujer, ocasionado principalmente por dos factores: el primero, al hecho que su carga familiar no ha variado en términos de responsabilidades, manejo, administración y ejecución de tareas, y dondela mujer ha asumido un doble rol, que ha significado una sobrecarga de trabajo aumentando los niveles deestrés, ansiedad y depresión, fenómenos que evidencian los efectos negativos del trabajo y del hogar sobrela salud mental dela mujer trabajadora (Blanco y Feldman, 2000) y, el segundo, la exposición a riesgos de distinta índole derivados de las condiciones en que desarrolla su actividad de trabajo y que no consideran las condiciones físicas propias del sexo (Servicio Nacional de la Mujer, SERNAM, 1992).

Estas características sociales, biológicas y laborales diferenciadas en función del sexo, hacen imprescindible abordar en forma conjunta las dimensiones salud, mujer y trabajo, debiendo incorporarse la perspectiva de gé nero en el análisis de las relaciones laborales y en el binomio condiciones de trabajo y salud (Brunel y Moreno, 2004). El análisis de los problemas de salud desde esta perspectiva, debe incorporar aquellas derivadas de las causas que expliquen la salud enfermedad (ángulo biológico), la construcción histórica de los géneros (ángulo psicosocial) y las diferentes nociones de los problemas asociados a la salud de la mujer y el trabajo doméstico como parte fundamental de la vida cotidiana de las trabajadoras y su doble jornada (ángulo sociohistórico) (Yanes, 2003; Garduño, 2001; Jaramillo y Uribe, 1999).

Sin embargo, los problemas de salud ocupacional de la mujer han sido poco o insuficientemente estudiados o deliberadamente evadidos, afirmación coincidente con el informe presentado, en 1987, por el grupo de trabajo para el Desarrollo de la Investigación sobrela M ujer en el Trabajo, reunido en Washington por la Organización Panamericana de la Salud (OPS), el cual destaca que "la producción científica en materia de investigaciones sobre la mujer en el trabajo ha sido escasa y se ha sesgado hacia el análisis de aspectos biotécnicos, morbilidad, esfera reproductiva y embarazo, refiriendo en sus estudios los factoresdeterminantes demanera aislada" (Acevedo, 1995). Por otra parte, el análisis de la situación actual dela salud delos trabajadoresrealizados por la OPS/OMS (Organización M undial de la Salud) para la región de las Américas en 1990, reconoce la existencia de grandes inequidades económicas y sociales en el sector laboral, siendo las mujeres las que trabajan en condiciones más precarias que los hombres, recibiendo sólo el $71 \%$ de la remuneración de los hombres, doble carga de trabajo, lo que la exponea mayores riesgo en salud escasamente abordados (OM S, 1999).

En Chileestetema emergenteno es enfrentado o lo es parcialmente por el sector salud, estimándose que la rápida incorporación de la mujer en la fuerza de trabajo, usualmente asociada al proceso de industrialización y urbanización, las ha afectado en forma particular, ya sea por falta de protección en salud, su doble filiación de trabajo (SERNAM , 2002), responsabilidad familiar y la falta de diseños y adaptaciones de los puestos de trabajo a su especificidad de género (Naveillán y cols., 2002). 
Investigaciones realizadas sobre la inserción laboral femenina en Chile muestran un aumento sostenido dela tasa departicipación femenina en la fuerza de trabajo, así mientras en 1970 el porcentaje de participación femenina era de $24,1 \%$, el año 2002 aumenta a un 35,03\% (Instituto Nacional de Estadísticas, INE, 2002), lo cual no necesariamente ha ido acompañado por un respaldo en la legislación, cuyas leyes de salud laboral no han incorporado medidas relativas a la especificidad delas mujeres acordes a esteincremento (SERNAM , 1992). Por otra parte, la reconversión productivaylainnovación tecnológica muestran que los procesos resultantes de las actividades laborales pueden afectar a las mujeres en forma directa o indirecta, ya sea por riesgo de accidentes en los lugares de trabajo, de contraer enfermedades profesionales, de sufrir intoxicaciones agudas o crónicas por compuestos químicos, de contraer o agravar enfermedades comunes por las condiciones de trabajo: sistema deturnos, a la intemperie, en lugares hacinados, lugares fríos o húmedos y riesgos ergonómicos por malas adaptaciones de los puestos de trabajo o instalaciones precarias (SERNAM, 1992).

La presente investigación intenta aportar nuevos antecedentes sobrela problemática de salud de la mujer trabajadora de residencia habitual en las comunas de jurisdicción del Servicio de Salud Concepción (INE, 2002), todas incorporadas al mundo laboral y definidas por grupos de edad donde la problemática de salud y sus riesgos son diferentes (Rodríguez, 1994).

Se pondrá especial énfasis en los aspectos, tanto de morbilidad asociados a grupos de patologías (CIE 10, 1995), magnitud y trascendencia, como demográficos vinculados a la edad, ubicación geográfica, tipo de actividad y ocupación (INE, 2002) y de salud, referidos principalmente a días y tipo de licencias médicas (M inisterio de Salud, M INSAL, 1984), estacionalidad, recuperabilidad y especialidad, describiendo para cada uno de ellos los principales aspectos vinculados a la pro- blemática del estudio. En resumen, el presentetrabajo deinvestigación actualizará y complementará los estudios existentes a objeto de conocer y entender desde una perspectiva de género si los problemas de salud ocupacional de las mujeres de las comunas en estudio adoptan formas diferentes en las distintas etapas de su vida y en las distintas ubicaciones sociales y comunales, de manera tal de construir un perfil actualizado de la morbilidad ocupacional de este importante grupo laboral, todo ello con el propósito de sugerir estrategias y medidas de enfermería cuya labor puede extenderse, ya sea tanto en la prevención de la patologías, la atención de enfermería en domicilio y lugares de trabajo, la rehabilitación, la orientación en salud, la consejería para disminución del estrés y adaptación al trabajo y el hacer visible los problemas y la participación en la promoción de leyes que apoyen en lo laboral a la mujeres, lo cual contribuirá a una mejor salud en el trabajo y el mejoramiento dela calidad de vida dela mujer trabajadora.

\section{MATERIALESY MÉTODO}

Tipo de estudio: La presente investigación corresponde a un estudio de tipo descriptivo y correlacional.

Universo: Como universo de este estudio se consideró a la totalidad de las mujeres trabajadoras que durante el año 2003 hicieron uso de licencias médicas por morbilidad y que residen y trabajan en las comunas de jurisdicción del Servicio de Salud Concepción: Comunas de Concepción, Chiguayante, San Pedro, Hualqui, Santa Juana, Florida, Coronel y Lota. Para efectos de la investigación se trabaja con el universo del estudio equivalente a un total de 28.750 licencias médicas emitidas, bajo el supuesto que cada licencia mé dica representa un episodio de morbilidad de la mujer trabajadora cuya complejidad es condicionante de la emisión de una licencia 
médica, no significando que cada licencia representa una mujer a nivel individual sino cada licencia como representativa de un episodio de morbilidad. Se excluyen las mujeres con licencias médicas de tipo maternal o por hijos enfermos menores de 1 año.

Unidad deanálisis: Las licencias médicas pertenecientes a las mujeres trabajadoras, extendidas por causas demorbilidad independientes del número de episodios, e incluidas en las base de datos de la Comisión de M edicina Preventiva e Invalidez, COM PIN, del Servicio de Salud Concepción correspondientes al año 2003.

Recolección de datos: Los datos fueron obtenidos a través de la información proveniente de las licencias médicas extendidas a las mujeres trabajadoras durante el año 2003, tabuladas eincluidas en las bases de datos D-BASE delicencias médicas de la oficina de COM PIN pertenecienteal Servicio deSalud Concepción.

Procesamiento de datos: Efectuados los procesos de validación e integración de los datos, se utilizó el programa estadístico SPSS, el cual permitió organizar estadísticamente los datos del estudio y efectuar los análisis uni y bivariados pertinentes.

Para la exploración y análisis de cada variableseutilizaron técnicas deestadística descriptiva dependientes de la naturaleza de la variable. Para el análisis inferencial se consideró como la variable dependiente del estudio las causas de morbilidad, siendo los predictores o variables independientes seleccionadas desde dos dominios 0 áreas generales: un conjunto decaracterísticas sociodemográficas de nivel individual que describen a las mujeres trabajadoras: comuna de residencia habitual, actividad económica, ocupación, edad, y un conjunto de variables denominadas de salud referidas a días de licencias mé dicas, estacionalidad, tipo de licencias médicas, recuperabilidad y especialidad. Desde el punto de vista metodológico, la comparación entregrupos sehizo con el análisis de varianza (AN OVA), las pruebas de contrastes post hoc HDS (Honestly Significant Difference) de Tukey y la medida defuerza de asociación eta cuadrado. El análisis de asociación entre variables se efectuó mediante la prueba de $\mathrm{X}^{2}$ reforzada por los coeficientes de asociación $V$ deCramer y medidas tipo Lambda. Todos con un nivel de significación estadística de 0.05 .

\section{RESULTADOS}

El análisis global de la magnitud y trascendencia de la fuerza laboral femenina y su relación con licencias médicas extendidas muestra que la tasa promedio de participación femenina en la fuerza detrabajo según comunas de residenciaes deun $37 \%$, con unatasa máxima de $40 \%$ para la comuna de Concepción y una mínima de $21 \%$ para la comuna de Florida, la primera de predominio urbano y la segunda de predominio rural. Respecto a la razón licencias médicas extendidas / fuerza de trabajo, los índices se distribuyen en dos categorías: aquellas comunas con valores superioresa 0,50: SantaJuana, Lotay Coronel (por cada mujer ocupada se extienden sobre 0,50 licencias/año) y aquellas con valores entre 0,29 a 0,50 para el resto de las comunas.

\section{Características sociodemográficas de la población estudiada}

El análisis exploratorio univariado de las variables descritas en la Tabla № 1 destaca las siguientes características: 
M orbilidad dela mujer trabajadora Servicio de Salud Concepción, Chile/ A. Torres Y T. PARAVIC

Tabla 1. Estadísticas descriptivas de las variables sociodemográficas en estudio.

\begin{tabular}{|l|rl|r|l|r|l|r|}
\hline Estadísticos & Edad & IR Com. residencia & IR Actividad laboral & \multicolumn{2}{c|}{ IR ocupación } \\
\hline $\mathrm{n}$ & 28750 & Chiguayante & 12,45 & Agricultura & 0,31 & Ejecutivo & 0,80 \\
Lmax- Lmin & $15-85$ & Concepción & 37,51 & M inas & 0,14 & Profesor & 8,30 \\
Rango & 70 & Florida & 0,58 & Industria manuf. & 14,59 & otro profesional & 6,27 \\
M edia & 39,44 & Hualqui & 1,50 & Construcción & 0,61 & Técnico & 4,40 \\
M ediana & 38 & Santa Juana & 1,28 & Electricidad & 0,07 & Vendedor & 8,25 \\
Moda & 30 & Lota & 9,43 & Comercio & 20,06 & Administrativo & 17,49 \\
DS & 11,53 & Coronel & 25,54 & Transporte & 0,69 & Operario trabaj. & 22,26 \\
CV & 0,3 & San Pedro & 11,72 & Finanzas & 7,96 & Empleada casa & 5,14 \\
Asimetría & 0,313 & Total & 100,00 & Servicios est. & 42,05 & otros & 27,08 \\
P25 & 30 & & & Asesora hogar & 8,62 & ign & 0,01 \\
P75 & 49 & & & No espec. & 4,91 & & 100,00 \\
& & & & 100 & & \\
\hline
\end{tabular}

Fuente: Licencias M édicas, SSC, año 2003.

IR: Importancia relativa.

Edad: Se observa que la edad de más alta frecuencia dela población en estudio correspondea 30 años, con un $50 \%$ demujerescon más de 38 años y un promedio de 39,44 años, desviándose del valor promedio en 11,53 unidades, con un límite máximo de la distribución de 85 añosy un límitemínimo de 15 años. Dado el tamaño dela muestra, seconcluyeque dicha variable puedeser considerada normal.

En relación a la residencia de las mujeres solicitantes de licencia médica, los porcentajes observados corresponden al peso poblacional de la población trabajadora, y donde las zonas urbanas concentran el mayor porcentaje de mujeres con actividad laboral.

El análisis según actividad laboral muestra que el mayor porcentaje de las licencias emana de trabajadoras provenientes de los serviciosestatales ( $42,05 \%)$, seguidos por aquellas proveniente del sector comercio $(20,06 \%)$ eindustrias manufactureras ( $14,59 \%)$, todas áreas de desempeño predominantemente femenino.

Respecto a la ocupación la mayor frecuencia seobserva en operarias y trabajadoras $(22,26 \%)$, seguido deadministrativo $(17,49 \%)$ y otros no definidos o catal ogados como otros en la fuente de datos $(27,08 \%)$.

\section{Características de salud de la población estudiada}

LasTablas № 2, № 3 y № 4 resumen los principales estadísticos y frecuencias de cada una de las variables de salud consideradas en el estudio, destacándose que: Los días según licencia médica extendida muestran una distribución de gran amplitud con valores mínimos de 1 día y máximo de 93 días, siendo los 7 días el valor de mayor frecuencia, valor significativamente inferior al promedio de 11,66 días y a la mediana de 8 días observada.

Respecto a la estacionalidad, el mayor porcentaje de licencias corresponde a los meses de junio a agosto, con un peso relativo de alrededor del $10 \%$ por cada mes y un peso acumulado de $31 \%$ en relación al total de licencias analizadas.

El estudio del tipo de licencias médicas extendidas según definición institucional, muestra que en su mayoría obedecen a causas por enfermedades 0 accidentes no relacionados directamente con el trabajo (98,35\%), con valores mínimos para aquellos catalogados como accidentes de trabajo y enfermedades profesionales $(1,51 \%$ y $0,09 \%$, respectivamente). 
Tabla 2. Estadísticas descriptivas de las variables de salud en estudio.

\begin{tabular}{|c|c|c|c|c|c|c|c|}
\hline Estadísticos & Días de LM & \multicolumn{2}{|c|}{ IR Estacionalidad } & \multicolumn{2}{|c|}{ IR Tipo de Licencia médica } & \multicolumn{2}{|c|}{ IR Recuperabilidad } \\
\hline $\mathrm{n}$ & 28750 & Enero & 6,25 & Enf.o acc.no trabajo & 98,35 & Sí & 98,42 \\
\hline Lmax- Lmin & $1 d-93 d$ & Febrero & 6,13 & Prórroga med. prev & 0,05 & No & 1,58 \\
\hline Rango & 92 & M arzo & 8,28 & Acc. trabajo & 1,51 & & 100,00 \\
\hline Media & 11,66 & Abril & 8,63 & Enf. profesional & 0,09 & & \\
\hline Mediana & 8 & M ayo & 9,79 & & 100,00 & & \\
\hline Moda & 7 & Junio & 10,99 & & & & \\
\hline DS & 8,5 & Julio & 10,62 & & & & \\
\hline CV & 0,7 & Agosto & 9,30 & & & & \\
\hline Asimetría & 1,3 & Septiembre & 7,65 & & & & \\
\hline P25 & 5 & Octubre & 8,63 & & & & \\
\hline P75 & 15 & Noviembre & 7,65 & & & & \\
\hline & & Diciembre & 6,08 & & & & \\
\hline & & & 100,00 & & & & \\
\hline
\end{tabular}

Fuente: Licencias M édicas, SSC, año 2003.

IR: Importancia relativa.

Recuperabilidad: Del análisis realizado sólo el $1,58 \%$ de las licencias extendidas fueron catal ogadas como licencias por causas demorbilidad no recuperables.

La Tabla $\mathrm{N} 03$ muestra las especialidades médicas de mayor demanda y grupo de causasmás frecuentes respecto a las licencias analizadas, correspondiendo éstas a medicina general $(37,81 \%)$, seguida por psiquiatría $(18,95 \%)$ y traumatología (11,62\%), las que en su conjunto representan el $68,4 \%$ del total estudiado, y cuyos grupos de causa corresponden atrastornos del comportamiento ( $25,08 \%)$, seguidas de enfermedades osteomusculares $(20,38 \%)$ y sistema respiratorio $(18,07 \%)$, los que en conjunto representan el $63,5 \%$ de la morbilidad total, quesumadosa grupo decausas con valores porcentuales mayoresa $4 \%$, dan cuenta del $83 \%$ de la morbilidad total (traumatismos, digestivas, genitourinarias).

Tabla 3. Principales especialidades y grupos de patologías demandantes de licencias médicas.

\begin{tabular}{|c|c|c|c|}
\hline \multicolumn{2}{|c|}{ Especialidades de mayor demanda } & \multicolumn{2}{|c|}{ Grupo de causas más frecuentes } \\
\hline $\mathrm{N}$ total $=$ & 28750 & $N=$ & 28750 \\
\hline N parcial (6 esp.) & 25278 & N parcial (7 grupos) & 23979 \\
\hline & 0,88 & & 0,83 \\
\hline Especialidades & & Grupos de causas & \\
\hline Medicina general & 37,81 & Trast. del comportamiento & 25,08 \\
\hline Psiquiatría & 18,95 & Enf. osteomusculares & 20,36 \\
\hline Traumatología & 11,62 & Enf. sistema respiratorio & 18,07 \\
\hline Cirugía & 9,20 & Traumatismos y env. & 6,10 \\
\hline Medicina Interna & 5,44 & Infecciosas y parasitarias & 5,16 \\
\hline \multirow[t]{2}{*}{ Gineco-obtetricia } & 4,90 & Enf. digestivas & 4,45 \\
\hline & & Enf. genitourinarias & 4,17 \\
\hline
\end{tabular}

Fuente: Licencias M édicas, SSC, año 2003. 
En la Tabla $\mathrm{N}$ o 4 se presentan los grupos decausas específicas derivadas de los grandes grupos de causas clasificados. Analizados los valores porcentuales, destaca el alto porcentajedelossíndromes depresivosy ansiosos en el grupo delos trastornos del comportamiento $(61 \%)$, constituyendo ambas, las patologías específicas de mayor prevalencia en el total del universo estudiado. Le siguen en magnitud las alteraciones osteomusculares, donde los lumbagos y las enfermedades cervicobraquiales representan el $41 \%$ del peso relativo de este grupo. Para el resto de los grupos, las causas específicas de mayor prevalencia son aquellas relacionadas con bronquitis, esguinces, contusiones, diarreas de origen infeccioso, hepatitis, colelitiasis y cuyos porcentajes se observan en la tabla descrita.

En el análisis de la variable edad, según factores sociodemográficos y de salud, se encontraron las siguientes características diferenciales y niveles de significación estadística:
Edad y residencia: Para los grupos menores de20 años, las comunas que presentaron mayor número de licencias médicas por morbilidad fueron las comunas de Coronel $(34,4 \%)$, Concepción $(24,7 \%)$ y Lota $(23,4 \%)$, en los grupos de 20 a 44 años, las comunas de Concepción $(34,6 \%)$, Coronel $(27,6 \%)$ y San Pedro (11,0\%), en los grupos de 45 a 64 años, las comunas de Concepción (43\%), Coronel $(21,6 \%)$ y San Pedro $(13,4 \%)$ y por último para los grupos de 65 años y más las comunas de Concepción (48,4\%), Coronel $(18,6 \%)$ y Chiguayante $(12,6 \%)$

Los predictores sociodemográficosutilizados respecto a la residencia indican que comuna y edad se asocian ( $F=69.38,7$ g.l, $p<$ 0.000 ), siendo dicha relación bastante débil $\left(\eta^{2}=0.017\right)$, existiendo 3 grupos de comunas homogéneos: Comuna de Concepción: mayor edad de las demandantes, Lota y Hualqui menor edad y las restantes de mediana edad.

Tabla 4. Causas específicas de morbilidad más frecuentes.

\begin{tabular}{|c|c|c|c|c|c|c|}
\hline \multicolumn{3}{|c|}{ Grupo de causas más frecuentes } & Grupo & \multicolumn{3}{|c|}{ Causas específicas más frecuentes } \\
\hline $\begin{array}{c}\text { Grupo } \\
\text { I }\end{array}$ & $\begin{array}{c}\mathrm{N}= \\
\text { Trast. del comportamiento }\end{array}$ & $\begin{array}{r}28750 \\
N \\
7211\end{array}$ & I & $\begin{array}{l}\text { Causas Específicas } \\
\text { Depresión } \\
\text { Ansiedad } \\
\text { Aporte\% al grupo I }\end{array}$ & $\begin{array}{r}N \\
3465 \\
963 \\
4428\end{array}$ & $\begin{array}{r}\% \\
0,48 \\
0,13 \\
0,61\end{array}$ \\
\hline II & Enf. Osteomusculares & 5854 & II & $\begin{array}{l}\text { Lumbago } \\
\text { S. cervicobraquial } \\
\text { Aporte \% al grupo II }\end{array}$ & $\begin{array}{r}1958 \\
467 \\
2425\end{array}$ & $\begin{array}{l}0,33 \\
0,08 \\
0,41\end{array}$ \\
\hline III & Enf. Sistema respiratorio & 5196 & III & $\begin{array}{l}\text { Bronquitis } \\
\text { Laringobronquitis } \\
\text { Aporte \% al grupo III }\end{array}$ & $\begin{array}{r}1262 \\
287 \\
1549\end{array}$ & $\begin{array}{l}0,24 \\
0,06 \\
0,30\end{array}$ \\
\hline IV & Traumatismos y Env. & 1755 & IV & $\begin{array}{l}\text { Esguinces } \\
\text { Contusiones } \\
\text { Aporte \% al grupo IV }\end{array}$ & $\begin{array}{l}486 \\
258 \\
744\end{array}$ & $\begin{array}{l}0,28 \\
0,15 \\
0,42\end{array}$ \\
\hline V & Infecciosas y parasitarias & 1484 & $\mathrm{~V}$ & $\begin{array}{l}\text { Diarreas y gastroent. } \\
\text { Hepatitis } \\
\text { Aporte } \% \text { al grupo V }\end{array}$ & $\begin{array}{r}978 \\
48 \\
1026\end{array}$ & $\begin{array}{l}0,66 \\
0,03 \\
0,69\end{array}$ \\
\hline VI & Enf. Digestivas & 1280 & VI & Colelitiasis & 241 & 0,19 \\
\hline & & & & $\begin{array}{l}\text { Hernia inguinal } \\
\text { Aporte \% al grupo VI }\end{array}$ & $\begin{array}{l}161 \\
402\end{array}$ & $\begin{array}{l}0,13 \\
0,31\end{array}$ \\
\hline VII & $\begin{array}{c}\text { Enf. Genitourinarias } \\
\text { Total } \\
\text { IR }\end{array}$ & $\begin{array}{r}1199 \\
23979 \\
0,83\end{array}$ & & Infecciones urinarias & 292 & 0,24 \\
\hline
\end{tabular}

Fuente: Licencias M édicas, SSC, año 2003. 
Edad y actividad Laboral: El mayor porcentaje de las jóvenes menores de 20 años que demandaron licencias por morbilidad corresponden a trabajadoras de industrias manufactureras $(40,8 \%)$, y de actividades del comercio $(30,5 \%)$, mientras que en el grupo de mujeres de 20-44 años son trabajadoras preferentemente del área de servicios estatales $(32,4 \%)$, y actividades de comercio $(25,9 \%)$, fenómeno similar a lo observado en mujeres de 45-64 años y 65 años y más, mostrando una fuerte relación con al área de servicios estatales ( $60,6 \%$ y $74,9 \%$, respectivamente) y comercio $(8,9 \%$ y $9,1 \%)$.

El análisis relacional de ambas variables indica que actividad laboral y edad se asocian $(F=479.6,10 \mathrm{~g} . \mathrm{l}, \mathrm{p}<0.000)$, siendo esta relación bastante fuerte $\left(\eta^{2}=0.143\right)$, y en donde se destacan 3 grupos homogéneos: grupos vinculadosa agricultura y electricidad menor edad ( $m e d i a=30$ años), grupos vinculados a área estatal mayor edad (media = 43 años), el resto en edades intermedias.

Edad y ocupación: El análisis bivariado delas variables edad y ocupación muestra diferencias entre los grupos de estudio, así mientras en las mujeres menores de 20 años el mayor porcentaje de las mujeres que demandaron licencias correspondeal grupo de operariosy trabajadores (44,8\%), en los grupos de 20 a 44 y 45 a 64 años fueron mujeres cuya ocupación correspondió al rubro otros ( $28 \%$ ), y en el grupo de 65 años y más correspondea profesores con un $26,4 \%$.

El análisis relacional indica que existen diferencias significativas entreedad y ocupación $(F=280.6,9$ g.l, $p<0.000)$, siendo esta relación débil $\left(\eta^{2}=0.081\right)$.

Edad y tipo de licencias médicas - Recuperabilidad: Dado el peso delas licencias médicas extendidas por causas de enfermedad o accidentes no vinculados al trabajo (98\%), existe igual tendencia para todos los grupos deedad. I gual fenómeno ocurrecon la recuperabilidad.
Edad y especialidad: La relación edad y especialidad a la que fue demandada la licencia médica muestra que en los grupos de menores de 20 años, el 57,8\% demandó a la especialidad demedicina general, seguidas por un 9,1\% a las especialidades de ginecología y obstetricia y cirugía respectivamente, para las mujeres de 20 a 44 se mantiene como primera prioridad medicina general con un $39,5 \%$, seguidas por psiquiatría con un $21,8 \%$, situación similar para las mujeres de 45 a 64 años con un $34,4 \%$ y $13,9 \%$, respectivamente, mientras que para las mujeres mayores de 65 años se mantiene como especialidad de mayor demanda medicina general con un $30,2 \%$ seguidas de traumatología con un $22,3 \%$.

El análisis deANOVA para diferencias entre medias muestra asociaciones estadísticamentesignificativas $(F=77.9,21 \mathrm{~g} . \mathrm{l}, \mathrm{p}<0.000)$, siendo esta relación débil $\left(\eta^{2}=0.054\right)$.

Edad y grandes grupos de causas: La Tabla № 5 muestra la relación entre los grupos de causas e intervalos de edad, pudiéndose observar que para los grupos de menores de 20 años los grupos de mayor prevalencialo constituyen las enfermedades osteomusculares, enfermedades respiratorias y traumatismos; para las mujeres de 20 a 44 años, las enfermedades asociadas a trastornos del comportamiento y osteomusculares; para los grupos de 45 a 64 años las enfermedades osteomusculares, trastornos del comportamiento conjuntamentecon las respiratorias y por último para las mujeres de 65 años y más, las enfermedades osteomusculares, traumatismos y respiratorias.

El análisis bivariado muestra una asociación estadísticamente significativa entre ambas $(F=91.72,17$ g.l, $p<0.000)$, siendo esta relación débil $\left(\eta^{2}=0.051\right)$, denotando tres grupos homogéneos: el primero que afecta a mujeres menores de 30 años correspondiente a enfermedades derivadas del parto y puerperio, el segundo a mujeres mayores de 45 años relativo a enfermedades tumorales y de los órganos de los sentidos y el tercero el resto de las edades y grupos. 
Morbilidad de la mujer trabajadora Servicio de Salud Concepción, Chile/ A. Torres y T. PARAvic

Tabla 5. Tabla de contingencia Edad - Principales causas de morbilidad por grandes grupos de causas (\%).

\begin{tabular}{|ll|r|r|r|r|r|}
\hline \multicolumn{1}{|c|}{ Grupos de causas/ Edad } & 15 a 19 años & 20- 44 años & $45-64$ años & 65 y más & Total \\
\hline A00 & Enf. infecciosas y parasitarias & 10,4 & 5,5 & 4,2 & 4,4 & 5,2 \\
C00 & Tumores & 0,61 & 2,01 & 4,3 & 2,5 & 2,6 \\
F00 & Trastornos del comportamiento & 7,8 & 22,8 & 18,4 & 12,9 & 25,1 \\
I00 & Enf. circulatorias & 0 & 1,3 & 3,8 & 6,9 & 2,2 \\
J00 & Enf. respiratorias & 19,5 & 18,2 & 17,7 & 16,03 & 18,1 \\
K00 & Enf. digestivas & 3,2 & 4,4 & 4,5 & 2,5 & 4,45 \\
M 00 & Enf. osteomusculares & 22,7 & 17,8 & 25,1 & 25,4 & 20,4 \\
N00 & S. genitourinario & 3,8 & 4,7 & 3,2 & 1,6 & 4,2 \\
S00 & Traumatismos y Env & 14,9 & 5,4 & 6,9 & 16,3 & 6,1 \\
\hline & Total parcial & 82,91 & 82,11 & 88,1 & 88,53 & 88,35 \\
\hline & Otros grupos & 17,09 & 17,89 & 11,9 & 11,47 & 11,65 \\
\hline
\end{tabular}

Fuente: Licencias M édicas, SSC, año 2003.

Tabla 6. Tabla de Contingencia Principales causas de morbilidad por grandes grupos de causas - Actividad laboral( \%).

\begin{tabular}{|l|c|c|c|c|c|c|c|c|c|}
\hline Rama actividad & $\begin{array}{c}\text { Enf. } \\
\text { infecciosas }\end{array}$ & Tumores & $\begin{array}{c}\text { Tr. del } \\
\text { comportamiento }\end{array}$ & $\begin{array}{c}\text { Enf. Sist. } \\
\text { nervioso }\end{array}$ & $\begin{array}{c}\text { Enf. } \\
\text { circulatorias }\end{array}$ & $\begin{array}{c}\text { Enf. } \\
\text { respiratorias }\end{array}$ & $\begin{array}{c}\text { Enf. } \\
\text { osteomusc }\end{array}$ & $\begin{array}{c}\text { Enf. Genito } \\
\text { urinarias }\end{array}$ & $\begin{array}{c}\text { Trauma- } \\
\text { tismos yEnv. }\end{array}$ \\
\hline Agricultura & 6,82 & 3,41 & 20,45 & 0 & 0,05 & 6,82 & 14,77 & 5,68 & 14,77 \\
M inas & 0 & 12,2 & 41,46 & 0 & 0,02 & 4,88 & 19,51 & 0 & 2,44 \\
Ind. M anuf. & 3,86 & 1,98 & 25,53 & 2,38 & 1,87 & 3,65 & 28,3 & 4,39 & 5,51 \\
Construcción & 2,84 & 0 & 40,91 & 2,84 & 0,05 & 7,39 & 13,07 & 3,98 & 9,66 \\
Electricidad & 10,53 & 0 & 15,79 & 0 & 0,01 & 10,53 & 26,32 & 5,26 & 0 \\
Comercio & 4,53 & 1,98 & 35,29 & 1,92 & 2,92 & 4,04 & 18,09 & 3,73 & 5,62 \\
Transporte & 2,53 & 1,52 & 40,91 & 0 & 0,11 & 4,55 & 19,19 & 6,06 & 2,02 \\
Finanzas & 4,41 & 4,02 & 33,35 & 1,88 & 1,45 & 4,63 & 15,08 & 4,37 & 4,11 \\
Serv. Estatales & 6,71 & 2,11 & 20,31 & 2,95 & 9,71 & 4,54 & 19,42 & 3,81 & 5,95 \\
Serv. Domest & 4,12 & 3,96 & 21,88 & 2,3 & 1,49 & 4,88 & 20,83 & 5,25 & 6,62 \\
No esp. & 2,06 & 8,43 & 10,91 & 4,46 & 0,37 & 6,09 & 23,25 & 5,95 & 13,32 \\
\hline Total & 5,16 & 2,69 & 25,08 & 2,56 & 18,07 & 4,45 & 20,36 & 4,17 & 6,1 \\
\hline
\end{tabular}

Fuente: Licencias M édicas, SSC, año 2003.

Causas de morbilidad y actividad laboral: $\mathrm{La}$ Tabla $\mathrm{N}$ o 6 muestra la relación entre grandes causas de morbilidad y rama de la actividad laboral de desempeño, destacándose para todas lasáreas el predominio delas enfermedades asociadas atrastornos del comportamiento y enfermedades osteomusculares. Desagregadas por ramas, el sector agricultura muestra una mayor prevalencia de traumatismos; el sector minería, enfermedades decomportamiento; la industria manufacturera, enfermedades del tipo osteomuscular; el sector construcción, enfermedades del comportamiento; el sector electricidad, trastornos asociados a enfermedades osteomusculares; el sector co- mercio, enfermedades del comportamiento, al igual que el sector finanzas y transporte; el sector estatal, enfermedades del comportamiento seguidos por enfermedades osteomusculares y, por último, el servicio doméstico, con igual perfil que los servicios estatales con mayor prevalencia deenfermedades del comportamiento seguidos por enfermedades osteomusculares.

Por último el análisis de $X^{2}$ muestra diferencias estadísticamente significativas entre causas y ramas de actividad $\left(X^{2}=2237.3,170\right.$ gl $p<0.000$ ), aun cuando la relación es bastante débil ( $V$ de Cramer $=0.08$ ). 
Causas demorbilidad y comunas deresidencia: El análisis de las causas de morbilidad según comunas deresidencia, no revela diferencias significativas en cuanto a causas de mayor importancia relativa (trastornos del comportamiento, osteomusculares), aun cuando la prueba de $\mathrm{X} 2$ resulta significativa ( $\left(\mathrm{X}^{2}=\right.$ $788.2,119 \mathrm{gl}, \mathrm{p}<0.000)$, esta relación es débil ( $\mathrm{V}$ de $\mathrm{Cramer}=0.06$ ).

Causas de morbilidad y enfermedades profesionales: Destacan como principales causas los traumatismos (82,3\%) y las osteomusculares (15,9\%).

Causas demorbilidad y accidentes del trabajo: Destacan las enfermedades osteomusculares y factores no especificados que influyen en el estado de salud ( $46 \%$ y $36 \%$, respectivamente).

\section{IV.DISCUSIÓN}

El análisis estadístico deesteestudio ha resultado valioso por los datos obtenidos, lo que es particularmente importante, al considerar que la morbilidad que presenta la mujer trabajadora es un área insuficientemente explorada a nivel de salud.

El análisis exploratorio muestra la magnitud de la morbilidad de la mujer diferenciada por comunas, llama la atención la razón licencias médicas/mujer ocupada delas comunas de Coronel y Lota, I c cual vinculada a las causas de morbilidad no muestra diferencias significativas con el resto delascomunas, siendo predominantes en todas ellas los trastornos del comportamiento y enfermedades osteomusculares, ambos asociados a depresión, ansiedad, lumbagos, todos fenómenos con gran influencia ambiental (Díaz, 1992). Esnecesario hacer notar la necesidad de intervención particularmenteen estas comunas, dado el fuerte impacto social que tienen estas patologías.

El análisis por grupos de edad destaca que la morbilidad laboral esun fenómeno queatraviesa todas las edades (González y Ferrechio, 1993), siendo las mujeres de mayor edad las más afectadas en las comunas de Concepción, y las de menor edad en las comunas de Lotay Hualqui, probablemente debido a la estructura poblacional de las mismas. Al asociar la edad a la actividad laboral se observa que las másjóvenes se dedican preferentementea actividades relacionadas a la industria manufacturera y comercio, siendo trabajadoras u operarias y donde las enfermedades osteomusculares, respiratorias y traumatismosadquieren mayor prevalencia, lo cual es concordante con lo encontrado en la bibliografía, donde los riesgos en dichasáreas por sobreesfuerzos posturales, riesgos ergonómicos, exposición al frío o lugares húmedos, riesgos de caídas, algias posturales, tensosinovitis son predisponentes para la prevalencia de estas patologías (SERNAM, 1992).

Por otra parte, las mujeres mayores de 20 años se desempeñan preferentemente en servicios estatales y comercio con ocupaciones mayoritariamente de tipo administrativas y deservicio (profesores, otros profesionales) y donde las patologías prevalentes se asocian a trastornos del comportamiento, osteomusculares, traumatismos y respiratorias, lo cual se correlaciona directamente con los riesgos de estas áreas, ya sea riesgos de accidentes por sobreesfuerzo, estrés, depresión, neurosis, enfermedades posturales, síndromes por uso excesivo deextremidades superiores, enfermedades respiratorias, enfermedades posturales, enfermedades cardiovasculares (sedentarismo, estrés), síndrome de burn-out (Blanco, 2000; Yanes, 2003).

Lo precedente se confirma al estudiar la relación causas de morbilidad y rama de la actividad laboral, dondecada uno delossectores destaca causas demorbilidad correlacionados con los riesgos definidos por la literatura (M artínez y Blanco, 2003; Borges, 1998; Rodríguez, 1994), así vemos que para el sector agrícola la patología demayor prevalencia son los traumatismos, lugar donde precisamente 
los mayores riesgos están vinculados a accidentes por sobreesfuerzo posturales y por manejo de equipos y materiales, mientras que para el resto de los sectores las patologías prevalentes son las relacionadas con el comportamiento, osteomusculares, respiratorias, traumatismos coincidentes con los riesgos de lossectores deservicios: riesgos de accidentes variados por sobreesfuerzo y uso de materiales, estrés, neurosis, enfermedades respiratorias y digestivas y riesgos del trabajo administrativo, salud, educación: Síndromes por uso excesivo de extremidad superior (SUEDES) y enfermedades posturales, enfermedades cardiovasculares (sedentarismo, estrés), síndrome de burn-out.

Llama particularmente la atención la alta prevalencia de las causas asociadas a trastornos del comportamiento: depresión, ansiedad, motivadas probablemente por la sobrecarga de trabajo (doble jornada), lo que aumenta los niveles de estrés, ansiedad, depresión evidenciados en la literatura. Por otra parte, la falta de diseños y adaptaciones de los puestos de trabajo a su especificidad de género, las condiciones precarias donde se desarrollan determinan el alto número de enfermedades osteomusculares encontrados y donde los lumbagos son causa preponderante(Garduño, 2001; Jaramillo y Uribe, 1999).

Por último, los hallazgos dela investigación confirman lallamada"visión reduccionista del trabajo y salud" (Díaz, 1992), ya que todas las causas encontradas son catalogadas como enfermedades 0 accidentes no del trabajo; sin embargo, al hacer un análisis profundo de ellas, vemos su gran asociación a la actividad laboral, fenómeno que es confirmado al relacionar los riesgos descritos con las patologías encontradas.

El profesional de enfermería debe utilizar este conocimiento para ayudar a formar una nueva concepción de la salud de la mujer, incorporando conceptos que reconceptualizen la salud y el proceso salud enfermedad, especialmente a nivel de salud laboral, adoptar un enfoquepreventivo en la mujer que previlegie el autocuidado (Jaramillo y Uribe, 1999), proporcionar un mejor conocimiento delos riesgos y patologías prevalentes, facilitando su adaptación a las condiciones laborales y mejorando su entorno laboral; debe comprender que lo que les suceda a ella afectaráa su núcleo familiar y por ende a la sociedad, sobre todo reconociendo que las causas de morbilidad tienen un gran componente de tipo social.

\section{V.CONCLUSIONES}

Al finalizar este estudio sobre morbilidad de la mujer trabajadora que reside en el área de jurisdicción del Servicio deSalud Concepción en las mujeres, se ha llegado a las siguientes conclusiones:

- Que las causas de morbilidad de la mujer trabajadora están directamenterelacionadas con los riesgos establecidos según actividad.

- Que la morbilidad se vincula directamente con la edad y áreas de desempeño.

- Que existe una subestimación de la misma, no reconociéndola como causas del trabajo.

- Que se hace evidente el peso que tiene el componente social y el rol dela mujer otorgado por la sociedad, exponiéndola a una sobrecarga, con un doble rol y exposición a riesgos de distintaíndoleque setraducen en la presencia de patologías encontradas en la investigación.

\section{REFERENCIAS BIBLIOGRÁFICAS}

Acevedo, D. (1995). La investigación de los problemas de salud de la mujer trabajadora. Rev. Salud de los trabajadores 3, 27-34.

Blanco, G.; Feldman, L. (2000). Responsabilidades en el hogar y salud dela mujer trabajadora. Rev. Salud Pública de M éxico 42, 217-224.

Borges, A. (1998). Personal de enfermería: Condiciones de trabajo de alto riesgo. Rev. Salud delos Trabajadores, 6,113-118.

Bravo, R. (2004). Mujeres chilenas. Tendencias en la última década. Censo 1992-2002. Sernam 1,121189. 
Brunel, S.; M oreno, N . (2004). Guía para la mejora de las condiciones de salud y trabajo de las mujeres ( 1 a Ed.). M adrid. Secretaría Confederal de la Mujer deCC.0O.

Díaz, X. (1992). La dimensión de género en la salud ocupacional en Chile. Sernam, 1, 27-36.

Garduño, M . (2001). Relación entre el trabajo doméstico y la salud de las mujeres. Rev. Salud de los Trabajadores 9, 35-42.

González, C.; Ferrechio, C. (1993). La situación de salud de la mujer en Chile. Sernam. 1, 11-25.

INE. Chile (2002). Censo. Resultados Población y Vivienda (Base de datos 2002. (Disco compacto; sistema operativo: Window 98; 48 M B Ram).

Jaramillo, D.; UribeT. (1999). La atención integral a la mujer: Un compromiso del personal de salud. Extraído el 2 de septiembre 2004, disponible en: tone.udea.edu.co/revista/marz99/atenmi

M artínez, M.; Blanco V. (2003). Trabajo y salud en niños, niñasy adolescentetrabajadores desector agrícola. Rev. Salud de los Trabajadores, 11, 5-18.
Ministerio de Salud Chile (1984). Reglamento de licencias médicas. Decreto Supremo $\mathrm{N} 03$.

Naveillan, C.; Vergara, M .; Kulcsweski, A.; García, R. (2002). Salud de la mujer en Chile. Sernam.

OM S. OPS (1999). 124 Sesión Comitéejecutivo. 21-25 junio 1999. Salud de los trabajadores en la región delas Américas. Washington D.C.

OPS (1995). Clasificación estadística Internacional de enfermedades y problemas relacionados con la salud. $10^{\circ}$ ed. Washington DC.

Rodríguez, A. (1994). Seguridad y salud de la mujer trabajadora frente a la reconversión industrial y el cambio tecnológico. Rev. Salud de los Trabajadores (2): 10-15.

SERNAM (1992). Salud laboral de la mujer en Chile. Documento de Trabajo № 22:3-65.

SERNAM (2002). Situación de las mujeres en el mundo laboral. D ocumento de trabajo № 77 . Santiago, Chile.

Yanes, L. (2003). El trabajo como determinante de la salud Rev. Salud de los Trabajadores (11) 1-22. 